Review Article

\title{
NANOTECHNOLOGY FOR DETECTION OF DISEASES CAUSED BY VIRUSES-CURRENT OVERVIEW
}

\author{
SRILAXMI CHIPPA ${ }^{1}$, VASANTI SUVARNA ${ }^{2 *}$ \\ ${ }^{1}$ SVKM's Dr. Bhanuben Nanavati College of Pharmacy, V. L. Mehta Road, Vile Parle (West), Mumbai 400056, Maharashtra, India, \\ ${ }^{2 *}$ Department of Pharmaceutical Chemistry, SVKM's Dr. Bhanuben Nanavati College of Pharmacy, V. L. Mehta Road, Vile Parle (West) \\ Mumbai 400056, Maharashtra, India \\ *Email: vasanti.suvarna@bncp.ac.in
}

Received: 24 Nov 2020, Revised and Accepted: 03 Feb 2021

\begin{abstract}
Nanotechnology is having a high impact on the development of a novel class of biosensors called nanobiosensors. This technology has utilized some extremely exciting elements for sensing phenomenon improvement. The utilization of nano-materials, nano-rods, nano-particles, nano-tubes have aided rapid, reliable reproducibility and its detection in a much better way. The unique properties of nanobiosensors and its varied applications have influenced biosensing research. Since longtime, nanobiosensors have been utilized worldwide for the diagnosis of diseases co-related with molecular detection of biomarkers. This paper highlights the use of such nanobiosensors for the detection of the virus, infections, fungal pathogens, Human Immunodeficiency Virus (HIV) related diseases such as Cardiovascular diseases (CDVs), Renal Arthritis (RA) through different techniques including electrochemical biosensing, optical biosensing, point of care-diagnostics etc.
\end{abstract}

Keywords: Nanobiosensor, Disease diagnosis, Voltammetry, Amperometry, Point of care testing

(C) 2021 The Authors. Published by Innovare Academic Sciences Pvt Ltd. This is an open access article under the CC BY license (https://creativecommons.org/licenses/by/4.0/) DOI: https://dx.doi.org/10.22159/ijpps.2021v13i4.40359. Journal homepage: https://innovareacademics.in/journals/index.php/ijpps.

\section{INTRODUCTION}

Viruses are the tiniest parasites which have the capability in affecting the physiological behaviour of the host in which they enter and infect. Several living species such as bacteria, plants, human beings and animals are the host of these viruses. Viruses are adaptable and their modes of action are varied; due to this greater number of deaths are occurring in the entire globe, especially because of the human immunodeficiency virus (HIV), Zika virus (ZIKs), influenza infections etc. The transmutation of the existing known viruses currently leads to epidemics, most probably when such mutation occurs at the single level of nucleic acid it may be fatal for the human population. Thus, early diagnosis of these viruses is of high importance for improving the patient's quality of life. Varied conventional approaches are available for the detection of viruses and their interaction in developing infections [1]. Biosensors and nano-bio sensors are one of the conventional methods for the detection of such viruses. Since a long time, the use of biosensors has been one of the fastest technologies due to its varied applications. A biosensor is defined as a device which is designed for detection and quantification of a biochemical molecule for example DNA sequence, antibodies, enzymes, immunological molecules or proteins. Biosensor device consists of three components viz; bioreceptor (analyzes the material), transducer (converts signal) and detector (gives response). Later, nanotechnology showed exciting ingredients for the improvement of the biosensing phenomenon, this technology developed a novel class of biosensors named as nano-biosensors. The high sensitivity of nano-bio sensors is one of the potential benefits for its detection over conventional methods [2].

Nanobiosensors are defined as the devices that are used for measurement of a biological or biochemical event by using any magnetic, optical or electronic technology through a compact probe. Due to the utilization of several nanomaterials such as nanoparticles, nanorods, nanotubes and nanowires this device enables faster detection and its reproducibility in a better way. High surface area nanomaterials are useful in producing nano-bio sensors with shorter response times and greater sensitivity. Selenium nanoparticles (SeNPs) are also being used for various therapeutic applications such as antioxidant, anticancer and antimicrobial etc [3]. The nanostructures present in nano-biosensors act as an intermediate layer in between physicochemical detector components and biological agents, with nanomaterials a transducer is combined to construct a biosensor. Nanobiosensors are classified in a varied diverse area for their sensing mechanism, such as optical, electrochemical, mechanical and calorimetric. Fig. 1 depicts the different variations and types of nanobiosensors. A nanobiosensor is majorly used to detect several biological agents, such as nucleic acids, pathogens, antibodies and metabolites. Nanobiosensors has varied applications ranging from the estimation and diagnosis in the in vivo aspects of the healthcare industry, environmentally monitoring toxicants, pollutants and physical aspects like heavy metal toxicity, humidity, etc. Recently in one of the articles it is revealed that nanovaccines and nanobots are also under consideration for the treatment of most of the diseases [4] Considering the advantages of these nanobiosensors, in current review, we will summarize different types of nanobiosensors developed so far for the detection of viral diseases [5].

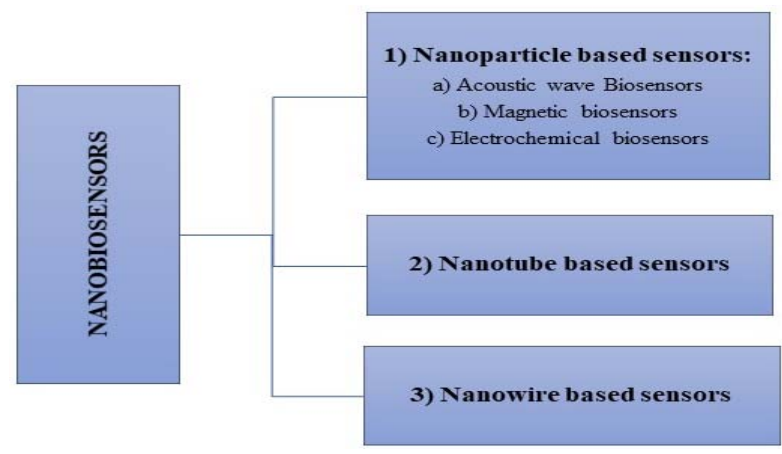

Fig. 1: Nanobiosensors types and variations

\section{Search criteria}

The selection of articles for the current review were searched by using keywords in certain specialized databases such as Elsevier (Science direct), Pubmed Medical subject headings (MeSH) and Medline. The keywords which were utilized are terms that were related to 
nanobiosensors and its diseases such as; "Nanobiosensors used for detection of viral diseases" OR "Viruses detected through nanobiosensors". Articles displayed on specialized databases were screened manually on basis of their title and abstract. Irrelevant and duplicate documents were excluded and those articles that met the inclusion and exclusion criteria were included in this study. Inclusion of these studies was based on the following criteria: (a) in a peer-reviewed journal it should be an original publication; (b) nanobiosensors should be utilized for the detection of diseases related to viruses. Only those articles which were written in English and published till now i. e; 2020 were reviewed. Exclusion criteria, were articles which are not applied to pharmaceutical field and those articles which were written in any language other than English, duplicates etc. After filtration and screening, 52 studies published between 1996-2020 met the inclusion criteria and these were included in the review. The selection strategy of review articles which were included is portrayed in the flow chart (fig. 2).

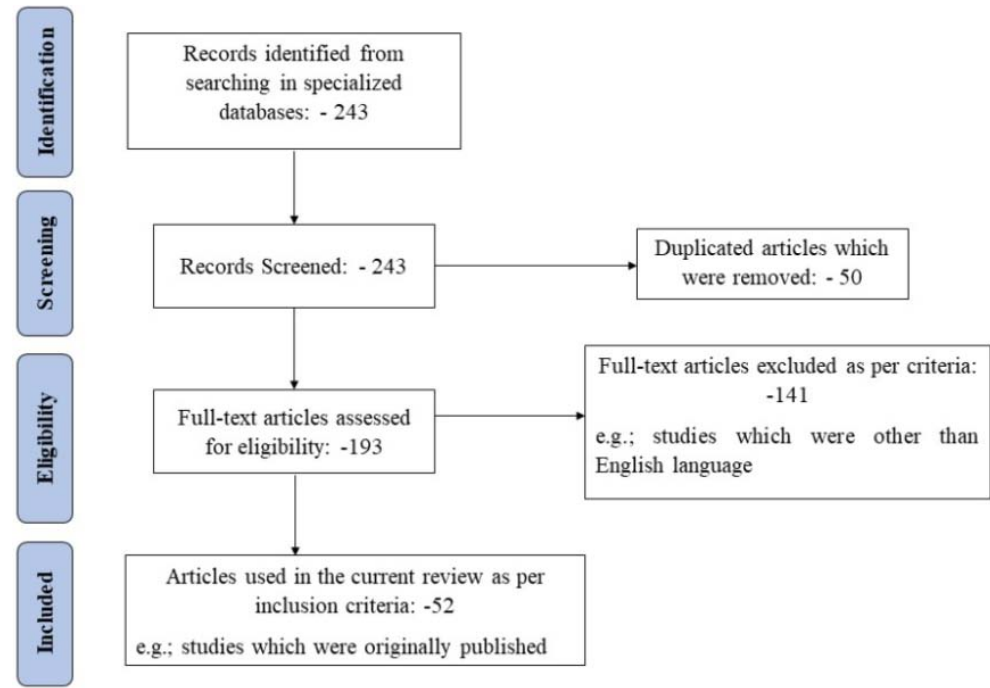

Fig. 2: Flow chart of the selection strategy of articles included in the review

\section{Overview of electrochemical nanobiosensors}

Biosensors based on nanomaterials have been utilized for the detection of biological molecules in the entire world. It is an evolving field where the interrogation of the electrochemical nanobiosensors is utilized in the form of impedance, current and potential. When there a is change in the signal response, the biological recognition event with high sensitivity and selectivity occurs. This type of biosensors is also utilized for the detection of glucose and are used with the combination of varied analytical techniques with their specificity of the desired analyte, such as conductometry, potentiometry, voltammetry etc. Due to the rapid detection and outstanding results through nanobiosensors these are used worldwide for detection of virus in disease diagnosis compared to conventional methods, such as RT-PCR, ELISA which are expensive and require more time [6]. Table 1 gives a glimpse of detection values, linearity range, nanomaterials used and detection method by Electrochemical nanobiosensors for diagnosis of diseases.

Table 1: Overview of electrochemical nanobiosensors in viral diseases

\begin{tabular}{|c|c|c|c|c|}
\hline Detection method & Nanomaterial & Limit of detection & Linear range & References \\
\hline EIS & Indium tin oxide electrodes & $2-3 \mathrm{ng} / \mathrm{ml}$ & $1-1000 \mathrm{ng} / \mathrm{ml}$ & [7] \\
\hline glip-T & $\begin{array}{l}\text { 1,6-Hexanedithiol and chitosan } \\
\text { stabilized gold nanoparticle }\end{array}$ & $0.32 \pm 0.01 \times 10-[14]$ & $1 \times 10^{-}[14]-1 \times 10^{-2} \mathrm{M}$ & [8] \\
\hline DNA hybridization & $\begin{array}{l}\text { Electrospun semi-conducting } \\
\text { Manganese (III) Oxide }\left(\mathrm{Mn}_{2} \mathrm{O}_{3}\right)\end{array}$ & $120 \times 10-[21] \mathrm{M}$ & - & [9] \\
\hline SWCNTs & Nanotubes & $10^{2} \mathrm{CFU} / \mathrm{ml}$ & $10^{2}-10[10] \mathrm{CFU} / \mathrm{ml}$ & {$[10]$} \\
\hline EIS & $\begin{array}{l}\text { Mercaptopropylphosphonic acid } \\
\text { functionalized copper doped zinc oxide } \\
\text { nanofibers }\end{array}$ & $6 \mathrm{ag} / \mathrm{ml}$ & $10 \mathrm{ag} / \mathrm{ml}-10 \mu \mathrm{g} / \mathrm{ml}$ & {$[11]$} \\
\hline Amperometry & $\begin{array}{l}\text { Silver graphene quantum dots } \\
\text { (Ag/GQDs) }\end{array}$ & $1 \mathrm{ZM}$ & $1 \mu \mathrm{M}$ to $1 \mathrm{ZM}$ & {$[12]$} \\
\hline Voltammetry & Nanoprobes & $4 \mathrm{pg} / \mathrm{ml}$ & $4-53 \mathrm{pg} / \mathrm{ml}$ & {$[13]$} \\
\hline $\begin{array}{l}\text { Nanoporous } \\
\text { membrane-based } \\
\text { nanobiosensor }\end{array}$ & 5' aminated DNA probes & $9.55 \times 10-[12] \mathrm{M}$ & $10^{-}[12]$ to $10^{-6} \mathrm{M}$ & [14] \\
\hline
\end{tabular}

EIS-Electrochemical impedance spectroscopy, SWCNTs-single-walled carbon nanotubes, CFU-colony forming unit, ag-attogram, $\mu$ g-microgram, ZMzepto molar, $\mu \mathrm{M}$-micro-molar

\section{Electrochemical methods}

Electrochemical nanobiosensors are the most common biosensors widely developed and utilized due to its several applications in agricultural, and especially in clinical applications and for industrial analysis. These biosensors generally provide several advantages ranging from high specificity of their biological recognition process, better specificity, low background noise, cost-effectiveness, rapid detection and better signal-to-noise ratio. Due to these varied applications and advantages, Electrochemical nanosensors are utilized for early detection of disease-related biomarkers [15]. Lin et al. (2018) designed a 3D nanostructured indium-tin-oxide (ITO) electrodes, immobilized it with toll-like receptor proteins (TLRs) for detection of the microbial pathogen components. Results revealed a linear dynamic 
range of $(>104)$ and magnitude lower detection limits of $(2-3 \mathrm{ng} / \mathrm{ml}$ of the microbial component) with 30 times better sensitivity for lipopolysaccharide and flagellin when compared with planar gold and ITO-based TLR sensors using TLR-based Electrochemical impedance spectroscopy (EIS) biosensors [7]. Bhatnagar et al., (2018) developed 1,6-Hexanedithiol and chitosan stabilized gold nanoparticle-mediated self-assembly of glip probes (glip-P) on gold electrode-based electrochemical biosensor for diagnosis of Invasive Aspergillosis, which is caused by Aspergillus fumigatus. The limit of detection of this biosensor was calculated to be as $0.32 \pm 0.01 \times 10-14$ (RSD $<5.2 \%)$ within a dynamic range of $1 \times 10-14-1 \times 10-2 \mathrm{M}$. [8] In another study, Tripathy et al., (2017) have detected dengue virus through nanofibres of Electrospun semi-conducting Manganese (III) Oxide (Mn203) based Electrochemical DNA nanobiosensor. The limit of detection was reported to be $120 \times 10-21$ M [9]. Yoo et al., (2017) developed a single-walled carbon nanotubes (SWCNTs)-based electrochemical nanobiosensor to detect microorganisms found in Asian dust events which is Bacillus subtilis. SWCNTs revealed a reliable and sensitive detection with a detection limit of $102 \mathrm{CFU} / \mathrm{ml}$ and a linear range of 102-1010 CFU/ml within 10 min of detection time [10]. Brince Paul et al., (2016) designed Mercaptopropylphosphonic acid functionalized copper doped zinc oxide nanofibers and synthesized these nanofibers through electrospinning technique to detect Plasmodium falcipuram histidine-rich protein-2. The biosensing showed detection limit of 6 attogram $/ \mathrm{ml}$, and detection range of $10 \mathrm{ag} / \mathrm{ml}-10 \mu \mathrm{g} / \mathrm{ml}$ with excellent sensitivity $28.5 \mathrm{~K} \Omega /(\mathrm{gm} / \mathrm{ml}) / \mathrm{cm} 2$ [11] Electrochemical nanobiosensors are also widely utilized for the detection of cancer [16].

\section{Amperometric methods}

Amperometric nanobiosensors have the ability to measure the current which is produced for determining the quantity of an analyte with fixed potential and measure the current through electrochemical oxidation or reduction at the working electrode. Recently the use of this biosensors is becoming more common [17]. In this study, Mobed et al. developed a novel paper-based genoassay for monitoring L. penumophila. Silver graphene quantum dots (Ag/GQDs) the ink was utilized for bioassay of L. penumophila. The quantification and detection limit of this platform was $1 Z \mathrm{ZM}$ and linear range of cDNA was $1 \mu \mathrm{M}$ to $1 \mathrm{ZM}$ via using chronoamperometry technique and results revealed that despite a simple structure $L$. penumophila has high sensitivity and specificity for the DNA based bioassay [12].

\section{Voltammetric methods}

Voltammetric nanobiosensors are most commonly used and available methods for ultrasensitive detection of various pathogens because of their high sensitivity, the ability of simultaneous quantification of targets, selectivity and cost-effectiveness. These nanobiosensors includes different methods such as linear sweep voltammetry (LSV), square wave voltammetry (SWV), differential pulse voltammetry (DPV) and cyclic voltammetry (CV). Nguyen et al., (2009) and colleagues have developed an electrochemical nanobiosensor based on membrane for sensing of inactivated West nile viral particle and West nile virus protein domain III (WNV-DIII) by utilizing anti-WNV-DIII immunoglobulin $M$ (IgM) as the biorecognition probe. The detection limit of this platform was 4 pg ml-1 via using voltametric detection method and high sensitivity was achieved [13].

\section{Membrane-based sensors}

Nanobiosensors based on membrane sensors is utilized worldwide in several biomedical applications. Because of the nature of nanospores, these are used as filters for the isolation of DNA, cells, RNA, bacteria, viruses and proteins detection. They have regular and uniform spaced nanochannels, which act as templates for the incorporation of nanosized materials ranging from metals, semiconductors, polymers, metals, and biomolecules which impart the novel biological and physicochemical characteristics to enhance the applications of membrane-based sensors. Membrane-based nanobiosensors have uniform size nanopores, high surface area, cost-effective, high aspect ratio and are easy to prepare [18]. Rai et al., (2012) designed ultrasensitive nanoporous membrane-based DNA biosensors using 5' aminated DNA probes and immobilized it on the alumina channel walls to detect the dengue virus (DENV). The detection limit of detection of this nanobiosensor was $9.55 \times 10-12$ $M$ with a linear range of over 6 order of magnitude i.e., 10-12 to 10-6 $M$ and high specificity [14]

\section{Overview of optical nanobiosensors}

Optical nanobiosensors are used widely due to its varied advantages over conventional techniques, which include high sensitivity, specificity, small size and less cost. Optical nanobiosensors are utilized in several fields, mainly healthcare, biotechnology, environment industry. Recently, the monitoring and diagnosis of varied diseases have undergone risky variations via optical biosensors. Fluorescence nanobiosensors are one of the most common optical methods used for detection of several pathogens/viruses because of their label-free detection capability, specificity, sensitivity, and cost-effectiveness [19]. Table 2 gives a glimpse of detection values, linearity range, nanomaterials used and detection method by optical nanobiosensors for diagnosis of diseases.

Table 2: Overview of optical nanobiosensors in viral diseases

\begin{tabular}{lllll}
\hline Detection method & Nanomaterial & Limit of detection & Linear range & References \\
\hline Fluorescence & CdTe QDs & $0.13 \mu \mathrm{m} \mathrm{ml}-1$ & - & {$[20]$} \\
Fluorescence & Oligonucleotide probes labeled with & $3 \times 10^{-8} \mathrm{M}$ & - & \\
& quantum dots and (AuNPs-Rh) & & & \\
Fluorescence & CdTe QDs & $0.2 \mathrm{nM}$ & 1.0 to $50.0 \mathrm{nM}$ & {$[22]$} \\
Fluorescence & Nanobeads & $10^{2}-10^{3} \mathrm{CFU} / \mathrm{ml}$ & $0-0.2 \mu \mathrm{M}$ & {$[23]$} \\
SPRi & AuNPs induced with QDs & $0.03 \mathrm{pg} / \mathrm{ml}$ and $0.4 \mathrm{pg} / \mathrm{ml}, 10 \mathrm{PFU} / \mathrm{ml}$ & - & {$[24]$} \\
Fluorescence & SiO -encapsulated alloyed CdZnSeS QDs & $8.2 \mathrm{copies} / \mathrm{ml}$ and $9.3 \mathrm{copies} / \mathrm{ml}$ & - & {$[25]$} \\
Fluorescence & FAIA & $220 \mathrm{pg} / \mathrm{ml}$ & $400 \mathrm{pg} / \mathrm{ml} \mathrm{to} 25 \mathrm{ng} / \mathrm{ml}$ & {$[26]$} \\
Microfluidic device & SERS & $0.01 \mathrm{IU} / \mathrm{ml}$ & $0.0125-60 \mathrm{IU} / \mathrm{ml}$ & {$[27]$} \\
Microfluidic chip & RT-LAMP & $1 \mathrm{copy} / \mu \mathrm{l}$ & - & {$[28]$} \\
\hline
\end{tabular}

AuNPs-Rh-rhodamine-immobilized gold nanoparticles, CdTe QDs-Cadmium-telluride quantum dots, CFU-colony forming unit, AuNPs-gold nanoparticles, FAIA-fluorescent anisotropic immunoassay, SERS-surface-enhanced Raman scattering, IU= international unit, RT-LAMP-reversetranscription loop-mediated isothermal amplification.

\section{Gold nanoparticles}

Over the past few decades, Gold nanoparticles (AuNPs) have been the subject of interest for research in biomedicine. AuNPs is also known as colloidal gold have novel diagnostic purposes or optical properties due to which it became popular worldwide in recent years for its applications in medicine. Nowadays, these AuNPs are utilized in the entire globe as point-of-care tests and novel testing strategies since it provides more sensitive, simpler, faster and costeffective assays to clinical laboratories [29]. In a study by Razmi et al., (2019) Tomato yellow leaf curl virus (TYLCV) genome in plants which are infected was detected using unmodified AuNPs and eliminated the need for detection equipment/PCR amplification [30]. Wang et al., (2017) utilized simple colorimetric detection 
method; for this he developed unmodified gold nanoparticles as colorimetric probes and detected Cucumber green mottle mosaic virus (CGMMV), which is transmitted by infected cucumber seeds, leaves and soil [31]. Shawky et al., (2010) also utilized colorimetric assay by developing unmodified AuNPs for direct detection of unamplified Hepatitis $\mathrm{C}$ virus (HCV) RNA, this assay was capable of detecting $50 \mathrm{HCV}$ copies/reaction [32]. Baetsen-Young et al., (2018) developed a novel unamplified genomic DNA (gDNA) nanosensor with the help of dextrin capped AuNPs to detect the causal agent of cucurbit downy mildew which is the unamplified DNA sequence from Pseudoperonospora cubensis, $2.94 \mathrm{fM}$ of pathogen DNA was detected [33]. Kaushal et al., (2019) designed the nanobiosensor based on gold nanorods (AuNRs) for the detection of foodborne bacteria due to its good electro-optical properties [34].

\section{Fluorescence}

Fluorescent nanomaterials are one of the promising approaches in optical nanobiosensors. These biosensors are also widely used as an alternative for conventional methods. In a recent study, Shokri et al., 2020 has detected citrus Tristeza virus by fluorescence anisotropic immunoassay through targeting the virus protein, CP25 to emit copper nanoclusters. The detection limit with assay was reported as $220 \mathrm{pg} / \mathrm{ml}$ within a linear range of $400 \mathrm{pg} / \mathrm{ml}$ to $25 \mathrm{ng} / \mathrm{ml}$ [26]. Similarly, Shojaei et al., (2016) reported a nanobiosensor based on fluorescence resonance-energy transfer (FRET) with the help of cadmium-telluride (CdTe) quantum dots (QDs) conjugated by a specific antibody against coat protein (CP) of CTV, and the CP were immobilized on the surface of gold nanoparticles (AuNPs) for determination of citrus Tristeza virus (CTV). The sensitive biosensor showed a detection limit of $0.13 \mu \mathrm{g} \mathrm{ml-1}$ and $93 \%, 94 \%$ of sensitivity, specificity respectively over enzyme-linked immunosorbent assay (ELISA) [20]. Sabzehparvar et al., (2019) developed FRET-based technique nano biosensor for detecting VP2 gene of infectious bursal disease virus utilizing two oligonucleotide probes labelled with quantum dots and rhodamine-immobilized gold nanoparticles (AuNPs-Rh) and the LOD value was estimated to be 3x10-8 M [21]. Shamsipur et al., (2017) also developed a nanobiosensor based on FRET by utilizing CdTe QDs, which is watersoluble and detected Human Papillomavirus 18 (HPV18). Results showed the detection limit of $0.2 \mathrm{nM}$ with an increase in DNA target concentration within the linearity range from 1.0 to $50.0 \mathrm{nM}$ [22]. In a study by Xu et al., (2017), a fluorescence nanobiosensor was designed by combining it with nanobead-based immunomagnetic separation for detection of bacterial pathogens in food products viz; E. coli, $L$. monocytogenes, S. Typhimurium. The detection limit for these bacterial pathogens was found to be $102,103,103 \mathrm{CFU} / \mathrm{ml}$ in lettuce, ground beef and shrimp, respectively [23]. Adegoke et al., (2016) designed an ultrasensitive QD fluorophore and exploited it as fluorescence signal generator. Further, modified silanization method for encapsulation of the thiol-capped QDs in a silica layer was modified and alloyed thioglycolic (TGA)-capped CdZnSeS QDs with high photoluminescence (PL) quantum yield (QY) value of $92 \%$ were synthesized to detect the norovirus. The authors reported a detection limit of 8.2 copies $/ \mathrm{ml}$ and $9.3 \mathrm{copies} / \mathrm{ml}$ in human serum and buffered respectively. Also, sensitivity was increased to 3-fold in comparison to norovirus RNA using TGA-capped CdZnSeS QD-MBs [25].

\section{SPR/SPRi}

AuNPs show a specialized phenomenon which is commonly called as Localized Surface Plasmon Resonance (LSPR) is responsible for intense red colour. SPR has a wide range of applications for diagnostic purposes; these nanobiosensors have become popular for its measurements in molecular interactions. An SPR biosensor was fabricated by Ming-Ju Chen, Kreuter et al., (1996) for the detection of insect pathogen-the baculovirus commonly called as Autographa californica multiple nuclear polyhedrosis virus (AcMNPV). Also, in another study, Boltovets et al., $(2004)$ detected tobacco mosaic virus (TMV) through SPR technique $[35,36]$. In another study, Takemura et al., (2017) developed a localized surface plasmon resonance induced immunofluorescence (SPRi) nano biosensor for detection of influenza virus H1N1 and H3N2. This biosensor was based on a gold nanoparticle-induced with quantum dot fluorescence signal. The LOD for H1N1 influenza virus was found to be $0.03 \mathrm{pg} / \mathrm{ml}$ and 0.4 $\mathrm{pg} / \mathrm{ml}$ in deionized water and human serum respectively, this showed high sensitivity. Whereas, H3N2 influenza virus which was clinically isolated reported the LOD value of $10 \mathrm{PFU} / \mathrm{ml}$ [24].

\section{Waveguide mode biosensors}

Waveguide biosensors are similar to the working procedure of surface plasmon resonance biosensing. The only difference in these both is the way of conducting measurements, these biosensors conduct measurements through waveguide mode. Maldonado et al., (2016) designed a bimodal waveguide (BiMW) based-photonic interferometer biosensor to detect Escherichia coli and Bacillus cereus infections caused in cirrhotic patients [37].

\section{Microfluidic devices}

Microfluidic devices are the new type of diagnostic methods which are sensitive, accurate, easy to use, and give rapid responses for dangerous viruses, including HBV, ZIKV, HIV and also in foodborne viruses. Microfluidic platforms depend upon the steps of miniaturization ranging from the preparation of the specimen to, bioreaction and further, it is detected into a unique system. The conventional techniques require much more time for the identification or detection of viruses, whereas these microfluidic devices consume less time for diagnosis and clinical treatment, which is vital for the patient's survival. Owing to the nature of these microfluidic kits, these might be advantageous in the localities with poor health facilities [38, 39]. Huang et al., (2017) designed a microfluidic chip insulated with air and detected varied pathogens related to pneumonia. The pathogens which where been detected are Mycoplasma pneumoniae, Staphylococcus aureus, and methicillinresistant $S$. aureus. $99.56 \%$ coincidence rate was determined, which reveals that the correct results were reported [40]. Kamińska et al., (2015) developed surface-enhanced Raman scattering (SERS) an ultrasensitive immunoassay with a new Raman reporter and unique SERS-active substrate and incorporated this in a microfluidic device to detect hepatitis B virus (HBV). The detection limit obtained was $0.01 \mathrm{IU} / \mathrm{ml}$ and high specificity was obtained [27]. Kaarj et al., (2018) detected Zika virus through a wax-printed paper microfluidic chip. This microfluidic chip was developed using reverse-transcription loop-mediated isothermal amplification (RT-LAMP). This platform showed the detection limit of 1 copy/ $\mu$ l and also proved that this technique can also be utilized for the identification of other flaviviruses, including dengue virus, chikungunya virus, also other viral pathogens that are quickly transmitted towards field-based diagnostics [28]. Dao et al., (2018) designed a new nanobiosensor based on isothermal amplification, which combines microfluidic enrichment by utilizing a concanavalin which is a functionalized microchannel consisting of asymmetric herringbone groove arrays. These nanobiosensors are utilized for rapid detection of pathogens so, with the help of this nanobiosensor Dao and his coworker's detected Salmonella enterica serotype Typhimurium bacteria which causes urinary tract infections. Due to the utilization of microfluidic enrichment the sensitivity increased by 1.76 orders of magnitude [41].

\section{Point of care testing (POCT)}

POCT is the cheap and effective medical diagnostic technique which are been utilized for the detection of varied infections or diseases caused through viruses such as, hepatitis, HIV and Influenza. POCT techniques are also used by patients at their bedside, which are classified into the following two types: the benchtop or the handled devices, which include HIV detection from salivary assay and checking of glucose levels. Some of the POC testings are based upon paper techniques which were applicable for the detection of viruses. This paper-based diagnosis is easy to use, inexpensive, and disposable, which makes it well adaptable technique. Over the course of time, for the detection of colorimetric substances, paperbased sensors were replaced through different methods, including fluorometric, chromatographic, electrochemical, microfluidics etc [42]. Zhang et al. and his co-workers designed a simple and effective detection method for infectious disease using quantum dots nanobeads (QDNBs) as the amplified signal indicators. This proved the detection of hepatitis B surface antigen (HBsAg) proteins and also improved the sensitivity of dot-blot immunoassay detection through amplification of fluorescent signals with quantum dot 
nanobeads [43]. Darbha et al. demonstrated that the HIV-1 viral DNA sequence can be screened by utilizing gold nanorods. The hyperRayleigh scattering (HRS) proved the efficient detection of HIV-1 virus DNA with an increase in intensity, sensitivity (100 picomolar) and selectivity [44]. Griffin $e$ t al. also utilized the HRS technique with gold nanoparticles for the detection of hepatitis $\mathrm{C}$ virus (HCV). The gold nanoparticles were conjugated with HCV ssRNA tagged along with rhodamine 6G. HCV ssRNA was detected and the selectivity reached a mismatch of single-base pair [45]. Chung et al., designed a nanoparticle system with a dual probe which was capable of phenotyping and detecting human pathogens. An assay of nanoparticle was designed specifically based on a sandwich hybridization technique which involved two probes of oligonucleotides to target the bacterial $16 \mathrm{~S}$ rRNAs for the detection of amplified targeted DNAs through the utilization of a miniaturized NMR device. The magneto-DNA platform was found, which allowed universal and specific detection of varied clinically relevant bacterial species, with sensitivity down to relevant bacteria. Further, they found the assay to be robust and rapid and simultaneously diagnosed a panel of 13 bacterial species in the clinical specimen within the time limit of $2 \mathrm{~h}$; also, a generic platform was formed for rapid identification of phenotype pathogens due to the varied applications [46]. In another study, Lee et al., and his colleagues developed a handheld diagnostic magnetic resonance (DMR) system for fast analysis using magnetic-nanoparticles as a proximity sensor for magnification of molecular interactions. It was found that DMR system is able to perform the measurements on biological samples which are unprocessed and can also be utilized for the characterization and detection of infectious agents ranging from bacteria, fungi, viruses etc. Later, they predicted that this DMR system when combined with the strategies of microfabrication it might be utilized as a portable, affordable, high-throughput POC nano diagnostics system for detection of significant infectious diseases in the future [47]. In yet another study by Liong et al., magnetic-nanoprobes have been utilized to develop the strategy of magnetic barcoding for the detection of Mycobacterium tuberculosis (MTB). PCR-amplified mycobacterial genes were captured on microspheres sequence-specifically, labelled by magnetic nanoprobes and were detected by NMR. The components were integrated into a fluidic cartridge for streamlined chip-operation, this platform was utilized for the detection of M. tuberculosis and for the identification of drug-resistant strains within $2.5 \mathrm{~h}$ from mechanically processed sputum samples. From the measurements of MTB-positive patient specimens, the clinical utility was demonstrated and the confirmation for the assay specificity was done clinically by non-MTB bacteria. Results demonstrated that a combination of magnetic barcode assay system with portable systems might be potential for becoming a sensitive, low-price and high throughput platform for infectious diseases point of carediagnostics [48]. Cihalova et al., also utilized magnetic barcode as a point of care diagnostics for the detection of most infectious fungal pathogens viz; Klebsiella pneumoniae, Staphylococcus aureus, methicillin-resistant Staphylococcus aureus and bacteria using fluorescent nanoparticle quantum dots (QDs). The results concluded that the method had the capability for the detection of infectious bacteria as low as the concentrations of $102 \mathrm{CFU} / \mathrm{ml}$ [49]. In a study by Inci et al., a unique nanoplasmic-based sensor was developed for the detection of HIV at different concentrations by using immobilized antibodies for capturing rapidly evolving viral subtypes selectively. Results demonstrated that detection of data analysis took $10 \mathrm{~min}$ and exhibited high sensitivity, specificity and repeatability of $98 \pm 39$ copies $/ \mathrm{ml}$. It also specified that the viral diseases detection directly from unprocessed blood samples of patients were feasible, showed a significant step towards POC tests at resource-constrained settings, hospitals etc. by enabling rapid detection, isolation, quantification and capture of viruses [50]. Kosaka et al. and his co-workers designed an immunoassay by the combination of both nano-mechanical properties of gold nanoparticles and opto-plasmonic transduction methods. By this immunoassay HIV-1, capsid antigen p24 in the human serum was detected. The limit of detection was reported to be $10-17 \mathrm{~g} / \mathrm{ml}$. and it was proved that this technology meets the demands to be produced en masse at a much lower cost, the capability for the miniaturization can be used as a point of care tests and also proved this platform to be an easily adaptive technique for diagnosis compared to Nucleic acid amplification tests (NAAT) [51]. Islam et al. developed a smart nanosensor utilizing graphene-based field effective transistors and detected HIV-related diseases, including renal arthritis (RA) and cardiovascular diseases (CVDs). Graphene was functionalized with aminoalkanes and was conjugated covalently with varied antibodies such as anti-p24 for HIV, anticyclic citrullinated peptide (anti-CCP) for RA and anticardiac troponin 1 (anti-cTn1) for CVDs via carbodiimide activation for detection of various biomarkers. Results concluded that high sensitivity with good linear curve was exhibited, the limit of detection for various antibodies were reported to be $10 \mathrm{fg} / \mathrm{ml}$ for cTn1 and CCP and $100 \mathrm{fg} / \mathrm{ml}$ for p24. Further, they concluded that the nanobiosensor based on graphene confirmed excellent performance and can be utilized for the on-site detection of HIV, Rheumatoid Arthritis and Cardiovascular diseases biomarkers in real samples [52]. Ng et al., proved the extensive utilization of POC system to detect HIV in saliva, leukocytosis in whole blood and plasma by utilizing mobile-based magneto-nano sensor arrays and magnetic nano-particles. This utilization of mobile-based magneto nano sensor device proved the ability to detect HIV in saliva and leukocytes in plasma quantitatively at a point of care within the assay time of 16 min with an accuracy of $90 \%, 80 \%$ respectively. Thus, they concluded that the portability, high sensitivity and ease of use of their nanodevice has the potential for becoming a low-cost and high throughput platform for point of care-diagnosis of HIV and early detection of diseases [53].

\section{Nucleic acids-and protein-based nanobiosensors}

Recently a newly based technique of nucleic acids-and protein-based nanobiosensors has been developed for detection of the novel emerging COVID-19 virus. The high specificity, selectivity and rapid biosensing activity of nanobiosensors have made it useful for the detection of specific proteins or nucleic acids of COVID-19.

\section{Immunoglobulin (Ig)-based nanobiosenors}

Immunoglobulin (Ig)-based nanobiosensors have also been utilized for the detection of newly emerging COVID-19 virus since our system can produce Ig in the face of COVID-19 utilizing nanobiosensor-based serological tests to determine specific antibodies which might be helpful.

Sharifi et al., recently showed these two reported diagnostic methods viz; nucleic acids-and protein-based nanobiosensors and Immunoglobulin (Ig)-based nanobiosensors for the detection of COVID-19 virus [54].

\section{Conclusion and future prospects}

Nanotechnology has significantly increased its demand in the development of biosensors and, has been proved useful for the detection of pathogens/viruses. Generally, the transduction mechanisms of these nanobiosensors have improved significantly due to the utilization of various nanoparticles for immobilization of enzymes, nanomaterials and nanostructures like those of quantum dots and hybrid nanostructures with varied functionalities. Unique advantages of these nanobiosensors over the assays of traditional methods such as low-price and high throughput platform, high sensitivity, specificity, rapid, reliable and real-time detection have made these nanobiosensors suitable for the detection of varied viruses. The use of nanobiosensors for POC testing has also enhanced sensitivity and became an appropriate signal amplification method. Some of the nanobiosensors have reached the limit of detection value in the picomolar and femtomolar range, due to which innovative strategies in the diagnosis of early-stage viruses is been provided in vivo and in vitro. However, the nanomaterials (NMs) biosafety must be observed in the utilization of nano biosensors for in vivo virus detection. Additionally, further efforts are required for the development of a nanosensing platform for detection of different virus-related diseases in vivo because most of the nanobiosensors have been employed for in vitro quantification. The more advancement and remarkable progress in the nanosensing platform with potential diagnostic features are expected to occur in future. 
Table 3: List of abbreviations

\begin{tabular}{|c|c|}
\hline HIV & Human immunodeficiency virus \\
\hline ZIKs & Zika virus \\
\hline DNA & Deoxyribonucleic acid \\
\hline RT-PCR & Reverse transcription polymerase chain reaction \\
\hline ELISA & Enzyme-linked Immunosorbent assay \\
\hline TLRs & Toll-like receptor proteins \\
\hline DENV & Dengue virus \\
\hline QDs & Quantum dots \\
\hline AuNPs & Gold nanoparticles \\
\hline LOD & Limit of Detection \\
\hline FRET & Fluorescence resonance-energy transfer \\
\hline SPR & Surface Plasmon Resonance \\
\hline SPRi & Surface plasmon resonance induced immunofluorescence \\
\hline SERS & Surface-enhanced Raman scattering \\
\hline POCT & Point of care testing \\
\hline QDNBs & Quantum dots nanobeads \\
\hline HBsAg & Hepatitis B surface antigen \\
\hline HRS & Hyper-Rayleigh scattering \\
\hline DMR & Diagnostic magnetic resonance \\
\hline NAAT & Nucleic acid amplification tests \\
\hline NMs & Nanomaterials \\
\hline CVDs & Cardiovascular diseases \\
\hline RA & Renal Arthritis \\
\hline
\end{tabular}

\section{RESPONSE TO COMMENTS}

The names of all the authors along with affiliations are included carefully in the main body of the manuscript.

Punctuation errors are rectified cautiously.

\section{FUNDING}

Nil

\section{AUTHORS CONTRIBUTIONS}

All the authors have contributed equally.

\section{CONFLICT OF INTERESTS}

\section{Declared none}

\section{REFERENCES}

1. Younis S. Nanosensors for the detection of viruses. Nanosensors for Smart Cities INC; 2020. p. 372-38.

2. Malik P. Nanobiosensors: Concepts and variations. ISRN Nanomater; 2013. p. 1-9.

3. Awanish Kumar, Kumar Suranjit Prasad. Biogenic selenium nanoparticles for their therapeutic application. Asian J Pharm Clin Res 2019;13:4-9.

4. Karthikeyan S, Laxmi Deepak Bhatlu M, Sukanya K, Jayan N. Nanovaccination: evolution and review. J Crit Rev 2020;7:971-5.

5. Prasad S. Nanobiosensors: the future for diagnosis of disease? Nanobiosensors Dis Diagnosis 2014;3:1-10.

6. Hammond JL, Formisano N, Estrela P, Carrara S, Tkac J. Electrochemical biosensors and nanobiosensors. Essays Biochem 2016;60:69-80.

7. Lin D, Harris KD, Chan NWC, Jemere AB. Nanostructured indium tin oxide electrodes immobilized with toll-like receptor proteins for label-free electrochemical detection of pathogen markers. Sensors Actuators B Chem 2018;257:324-30.

8. Bhatnagar I, Mahato K, Ealla KKR, Asthana A, Chandra P. Chitosan stabilized gold nanoparticle mediated self-assembled gliP nanobiosensor for diagnosis of Invasive Aspergillosis. Int J Biol Macromol 2018;110:449-56.

9. Tripathy S, Krishna Vanjari SR, Singh V, Swaminathan S, Singh SG. Electrospun manganese (III) oxide nanofiber based electrochemical DNA-nanobiosensor for zeptomolar detection of dengue consensus primer. Biosens Bioelectron 2017;90:378-87.

10. Yoo MS. Development of electrochemical biosensor for detection of pathogenic microorganism in Asian dust events. Chemosphere 2017;175:269-74.
11. Brince Paul K. A highly sensitive self assembled monolayer modified copper doped zinc oxide nanofiber interface for detection of Plasmodium falciparum histidine-rich protein-2: targeted towards rapid, early diagnosis of malaria. Biosens Bioelectron 2016;80:39-46.

12. Mobed A. Immobilization of ssDNA on the surface of silver nanoparticles-graphene quantum dots modified by gold nanoparticles towards biosensing of microorganism. Microchem J 2020;152:104-286.

13. Nguyen BTT. Membrane-based electrochemical nanobiosensor for the detection of virus. Anal Chem 2009;81:7226-34.

14. Rai V. Ultrasensitive cDNA detection of dengue virus RNA using electrochemical nanoporous membrane-based biosensor. PLoS One 2012;7:1-7.

15. Huang Y, Xu J, Liu J, Wang X, Chen B. Disease-related detection with electrochemical biosensors: a review. Sensors (Switzerland) 2017;17:1-30.

16. Sharifi M. Cancer diagnosis using nanomaterials based electrochemical nanobiosensors. Biosens Bioelectron 2019;126:773-84.

17. Perumal V, Hashim U. Advances in biosensors: principle, architecture and applications. J Appl Biomed 2014;12:1-15.

18. Van den Hurk R, Evoy S. A review of membrane-based biosensors for pathogen detection. Sensors (Switzerland) 2015;15:14045-78.

19. Ghorbani F, Abbaszadeh H, Dolatabadi JEN, Aghebati Maleki L, Yousefi M. Application of various optical and electrochemical aptasensors for detection of human prostate specific antigen: a review. Biosens Bioelectron 2019;142:111484.

20. Shojaei TR. Detection of citrus tristeza virus by using fluorescence resonance energy transfer-based biosensor. Spectrochim Acta Part A Mol Biomol Spectrosc 2016;169:216-22.

21. Sabzehparvar F, Rahmani Cherati T, Mohsenifar A, Roodbar Shojaei $\mathrm{T}$, Tabatabaei M. Immobilization of gold nanoparticles with rhodamine to enhance the fluorescence resonance energy transfer between quantum dots and rhodamine; new method for downstream sensing of infectious bursal disease virus. Spectrochim Acta Part A Mol Biomol Spectrosc 2019;212:173-9.

22. Shamsipur M. A highly sensitive quantum dots-DNA nanobiosensor based on fluorescence resonance energy transfer for rapid detection of nanomolar amounts of human papillomavirus 18. J Pharm Biomed Anal 2017;136:140-7.

23. $\mathrm{Xu}$ L. In-field detection of multiple pathogenic bacteria in food products using a portable fluorescent biosensing system. Food Control 2017;75:21-8.

24. Takemura K. Versatility of a localized surface plasmon resonance-based gold nanoparticle-alloyed quantum dot 
nanobiosensor for immunofluorescence detection of viruses. Biosens Bioelectron 2017;89:998-1005.

25. Adegoke O, Seo MW, Kato T, Kawahito S, Park EY. An ultrasensitive SiO2-encapsulated alloyed CdZnSeS quantum dot-molecular beacon nanobiosensor for norovirus. Biosens Bioelectron 2016;86:135-42.

26. Shokri E. Virus-directed synthesis of emitting copper nanoclusters as an approach to simple tracer preparation for the detection of Citrus Tristeza Virus through the fluorescence anisotropy immunoassay. Sensors Actuators B Chem 2020;321:128-634.

27. Kaminska A. Detection of hepatitis B virus antigen from human blood: SERS immunoassay in a microfluidic system. Biosens Bioelectron 2015;66:461-7.

28. Kaarj K, Akarapipad P, Yoon JY. Simpler, faster, and sensitive zika virus assay using smartphone detection of loop-mediated isothermal amplification on paper microfluidic chips. Sci Rep 2018;8:1-11.

29. Azzazy HME, Mansour MMH, Samir TM, Franco R. Gold nanoparticles in the clinical laboratory: principles of preparation and applications. Clin Chem Lab Med 2012;50:193-209.

30. Razmi A. Localized surface plasmon resonance biosensing of tomato yellow leaf curl virus. J Virol Methods 2019;267:1-7.

31. Wang L. Colorimetric detection of cucumber green mottle mosaic virus using unmodified gold nanoparticles as colorimetric probes. J Virol Methods 2017;243:113-9.

32. Shawky SM, Bald D, Azzazy HME. Direct detection of unamplified hepatitis $\mathrm{C}$ virus RNA using unmodified gold nanoparticles. Clin Biochem 2010;43:1163-8.

33. Baetsen Young AM. Direct colorimetric detection of unamplified pathogen DNA by dextrin-capped gold nanoparticles. Biosens Bioelectron 2018;101:29-36.

34. Kaushal S. Glycoconjugates coated gold nanorods based novel biosensor for optical detection and photothermal ablation of food borne bacteria. Sensors Actuators B Chem 2019;289:207-15.

35. Ming Ju Chen, Kreuter JYTK. Nanoparticles and microparticles for drug and vaccine delivery. J Anat 1996;189:503-5.

36. Boltovets PM. Detection of plant viruses using a surface plasmon resonance via complexing with specific antibodies. J Virol Methods 2004;121:101-6.

37. Maldonado J, Gonzalez Guerrero AB, Dominguez C, Lechuga LM. Label-free bimodal waveguide immunosensor for rapid diagnosis of bacterial infections in cirrhotic patients. Biosens Bioelectron 2016;85:310-6.

38. Chiu DT. Small but perfectly formed? successes, challenges, and opportunities for microfluidics in the chemical and biological sciences. Chem 2017;2:201-23.
39. Song J. Two-stage isothermal enzymatic amplification for concurrent multiplex molecular detection. Clin Chem 2017;63:714-22.

40. Huang G. A rapid, low-cost, and microfluidic chip-based system for parallel identification of multiple pathogens related to clinical pneumonia. Sci Rep 2017;7:1-10.

41. Dao TNT. Rapid and sensitive detection of salmonella based on microfluidic enrichment with a label-free nanobiosensing platform. Sensors Actuators B Chem 2018;262:588-94.

42. Martinez AW, Phillips ST, Whitesides GM. Diagnostics for the developing world microfluidic. Anal Chem 2010;82:3-10.

43. Zhang P, Lu H, Chen J, Han H, Ma W. Simple and sensitive detection of HBsAg by using a quantum dots nanobeads based dot-blot immunoassay. Theranostics 2014;4:307-15.

44. Darbha GK, Rai US, Singh AK, Ray PC. Gold-nanorod-based sensing of sequence specific HIV-1 virus DNA by using hyper-rayleigh scattering spectroscopy. Chem A Eur J 2008;14:3896-903.

45. Griffin J. Sequence-specific HCV RNA quantification using the size-dependent nonlinear optical properties of gold nanoparticles. Small 2009;5:839-45.

46. Chung HJ, Castro CM, Im H, Lee H, Weissleder R. A magnetoDNA nanoparticle system for target specific bacterial identification. Nat Nanotechnol 2013;8:369-75.

47. Lee H, Sun E, Ham D, Weissleder R. Chip-NMR biosensor for detection and molecular analysis of cells. Nat Med 2008;14:869-74.

48. Liong M. Magnetic barcode assay for genetic detection of pathogens. Nat Commun 2013;4:1752-9.

49. Cihalova K. Antibody-free detection of infectious bacteria using quantum dots-based barcode assay. J Pharm Biomed Anal 2017;134:325-32.

50. Inci F. Nanoplasmonic quantitative detection of intact viruses from unprocessed whole blood. ACS Nano 2013;7:4733-45.

51. Kosaka PM, Pini V, Calleja M, Tamayo J. Ultrasensitive detection of HIV-1 p24 antigen by a hybrid nanomechanicaloptoplasmonic platform with potential for detecting HIV-1 at first week after infection. PLoS One 2017;12:1-13.

52. Islam S. A smart nanosensor for the detection of human immunodeficiency virus and associated cardiovascular and arthritis diseases using functionalized graphene-based transistors. Biosens Bioelectron 2019;126:792-9.

53. Ng E, Yao C, Shultz TO, Ross Howe S, Wang SX. Magnetonanosensor smartphone platform for the detection of HIV and leukocytosis at point-of-care. Nanomed Nanotechnol Biol Med 2019;16:10-9.

54. Sharifi M. Rapid diagnostics of coronavirus disease 2019 in early stages using nanobiosensors: challenges and opportunities. Talanta 2021;223:121-704. 